\title{
ASPECTS REGARDING MISCANTHUS CUTTING PROCESS FEM SIMULATION AND SOME EXPERIMENTAL DATA
}

\author{
Georgiana Moiceanu, Gheorghe Voicu, Gigel Paraschiv, George Ipate \\ University Politehnica of Bucharest, Romania \\ moiceanugeorgiana@gmail.com,ghvoicu_2005@yahoo.com,paraschiv2005@yahoo.com, \\ puiuipate@yahoo.com
}

\begin{abstract}
A very important step in vegetal biomass processing is represented by the plant cutting process. There are many cutting methods/techniques all being different according to the knife characteristics. In order to have the optimum processing equipment, a modelling and simulation analysis is necessary, which can offer answers regarding the cutting process and the stress that takes place during cutting. In this paper cutting operation simulation was analyzed using miscanthus $x$ giganteus stalks. Simulation process was done by using the finite element method (FEM) with the help of ANSYS Multiphysics programme. For simulation, data regarding the plant physical properties as well as the knife properties were entered in the program. Also, for simulation, the theory that miscanthus stalks are formed out of two layers was agreed: miscanthus stalk shell with $E=1.9 \mathrm{GPa}$ and $v=0.29$ ( $E$ - elasticity module, $v$ - Poisson coefficient) and the plants core $E=100 \mathrm{MPa}$ and $v=0.29$. Thus, it was possible to create the plant geometry and the knife used for cutting. Shear stress applied in the simulation program had values of $100 \mathrm{~N}$ respectively $250 \mathrm{~N}$. The simulation process was accompanied by experimental research, the miscanthus stalk biomass was subjected to shear stress using type $\mathrm{V}$ plate with different opening angles. After testing the force - deformation curves resulted in the shear process. It was concluded that once the average diameter values and the stalk core shell thickness increased, the shear stress increased, the results obtained being in agreement with other experimental research done worldwide by researchers.
\end{abstract}

Keywords: simulation process, miscanthus biomass, cutting process, shear stress.

\section{Introduction}

Biomass formed out of vegetal material makes a useful renewable energy category in carbon dioxide reduction in nature and in the creation of an energy consumption environment mostly based on renewable energy. Because of the large dimensions of vegetal materials, the use of biomass is difficult, thus there is the need to apply several mechanical pretreatment processes. The most common mechanical pretreatment processes are material particle size reduction with the purpose to transform the material in order to obtain bio-diesel. According to scientists [1], the material size reduction process is a process that requires a high energy consumption. Taking into consideration that these crops and vegetal material are used much more frequently, the improvement of processing is needed in order to reduce the energy consumption, especially while harvesting biomass [2].

In other papers the authors tried to determine the optimal level of size reduction through a combination of modelling and experimental studies used for testing miscanthus and switchgrass. The experimental results had shown that specific energy consumption decreased according to a power law with increasing the particle size. The scientific article conclusion was that it is necessary to have an optimal miscanthus particle size range of about 4-6 mm is order to not increase the costs [3].

The grinding process was also the subject of another paper that created an optimization model so that this can be used in the densification process. Their biomass was wood and after experimental research and optimization of the model they concluded that in this case it is less convenient to bring the wood dimensions very small in order to store it [4]. For pellet production the particles should be less than $3.2 \mathrm{~mm} \mathrm{[5]} \mathrm{and} \mathrm{for} \mathrm{briquette} \mathrm{production} \mathrm{the} \mathrm{particle} \mathrm{size} \mathrm{should} \mathrm{be} \mathrm{in} \mathrm{the} \mathrm{range} \mathrm{of} 68 \mathrm{~mm}$ [6].

FEM analysis on the biomass chopping process was done in another scientific paper, which took into consideration different key parameters, like feeding rate, shell angle, distances of chopping and fixed blade. All these parameters were qualified by considering each influence on the power consumption and qualified rate. The material used for testing was wheat straws. After testing scientists concluded that the most important parameter is the feeding speed followed by the shell angle and distances of blades. Also, they said that the factor that had the highest influence on power consumption was the shell angle followed by the feeding rate [7]. Other scientists who also used DEM - FEM simulation chose to focus on modelling corn harvesting in order to give input regarding machine design and development. Tests had shown that the maize is locally compressed and pulled down, if the stalk rollers are spinning in opposite directions. Also, the scientists mentioned that by 
using the FEM analysis the stress distribution on cutting can be analyzed while biomass is harvested [8].

The scientists continued their research and made a comparative analysis of different geometrical structures of the discrete element method (DEM) for fibrous agricultural materials. They considered corn stalk as a model and divided it into parts. The stalk was divided by internodes and as pointed in the paper the corn stalk showed different mechanical characteristics for both tangential and axial directions. Their study proved that by using the FEM analysis different types of biomass can be subjected to analyze along with parts of the testing equipment. Also, they have shown that complex structures (materials) need more time to be analysed in order to give a significant result [9].

Another paper studied the connection that is created between the grass stalk and rotation mower by using DEM-FEM simulation, evidentiating the cutting speed, energy efficiency and the cutting quality [10]. Thus, considering the fact that the FEM analysis is a model that was used for simulation the harvesting process for different biomass types, in this paper our purpose was to simulate the cutting operation using miscanthus $\mathrm{x}$ giganteus stalks. Also, the simulation was accompanied by experimental research, the miscanthus stalk biomass being subjected to shear stress using type $\mathrm{V}$ plate with different opening angles.

\section{Materials and methods}

For better illustrating the result obtained in this paper the authors used the principles that apply to the cutting mechanism for lignocellulose plant stalks. Different ways for plant texture failure can occur during cutting, depending on the knife characteristics and the plant morphology. For stalk with a high moisture content and high knife cutting speed, the pressure in stalks is limited to an initial compression of the plant. A higher compression takes place for a higher speed, considering the knife sharpening edge and the cutting speed. Precompression before cutting with a gradual accumulation of force and the energy for precompression during cutting can form the energy consumption from 40 to $60 \%$ out of the total amount of the cutting energy consumed [11].

While the plant fibers are deviated from the cutting knife sharpening edge, the shear force of the material is mobilized to produce additional stress on fibers. These become large enough to make the fibers yield the stress applied by the knife. In Fig. 1, the typical form of the force - deformation curve, when a plant is cut by a knife and a counter knife, is presented. In section A compression takes place because the plant shell yields without the pressing force being high enough in order to cut the plant. After the stalk shell yields the compression and the cutting process continues in section B, where the highest pressing force is registered. In section $\mathrm{C}$ the material in sufficiently compressed and the cutting process continues until the end and the pressing force decreases rapidly, the knige cutting edge reaching the counter knife cutting edge. From the diagram it can be observed that the cutting energy in equal to the surface area below the plant cutting curve, which multiplied with the cutting frequency results in power.
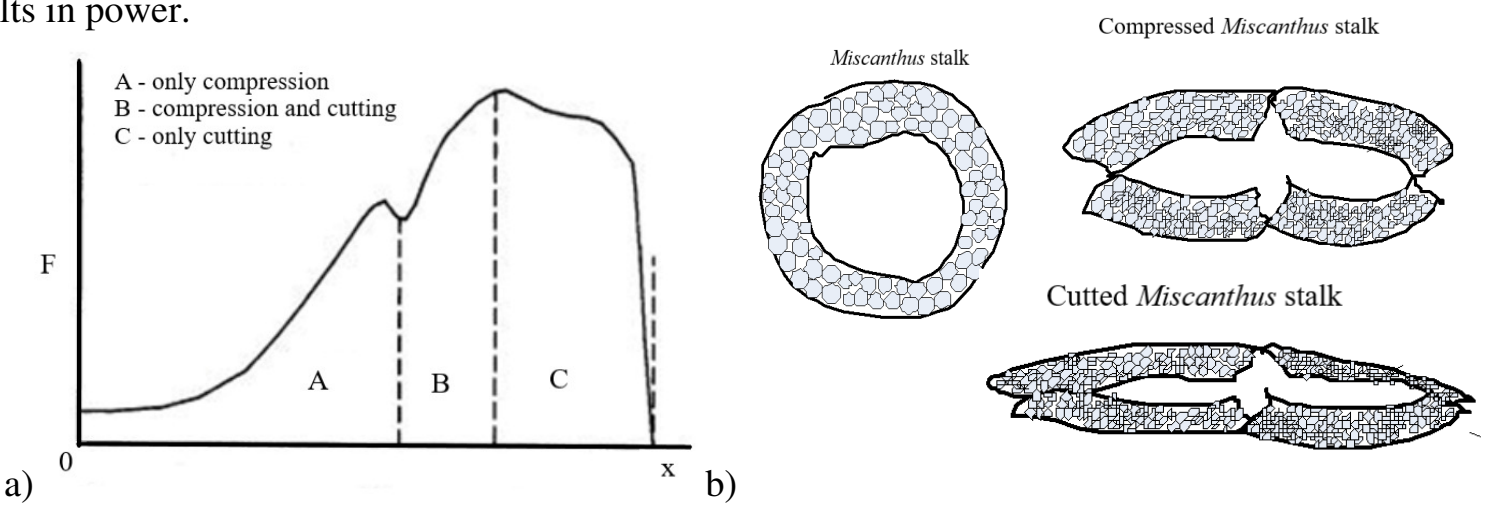

Fig. 1. Typical form of force - deformation curve and cutting phases: a - knife force cutting displacement curve for one cut; $b$ - the three phases of cutting plants with lignocellulose wall and soft core [11;12]) 
Before the experiments a miscanthus stalk cutting process simulation was realized using the FEM (finite element method) analysis. The program being used was ANSYS Multiphysics installed on a computer with AMD Turion 64 processor and an operating system Windows 7 Professional.

The 3D modelling cutting system had the main objective to simulate the way miscanthus stalk behaves during the cutting process. The first phaze of this modelling process was represented by the geometric model generation followed by the discretized model generation. Between these steps a simplification hypothesis was applied with which the model could be generated, steps like finding a simplification solution to reduce the model geometry to half considering the stalk symmetry with the purpose of making the numeric calculations simpler and the analysis time shorter. In order to realize the modelling process it was necessary to know the plant elasticity model and the Poisson coefficient, accepting the values $E=1.9 \mathrm{GPa}$ and $v=0.29$ for the stalk shell and $E=100 \mathrm{MPa}, v=0.29$ for the plant core. The stalk model geometry realized with SolidWorks is presented in Fig. 2.

a)

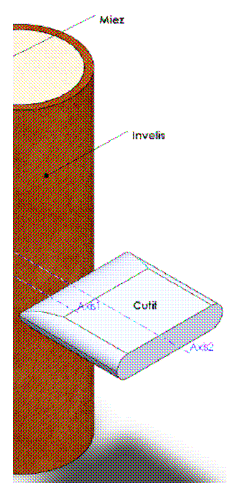

b)

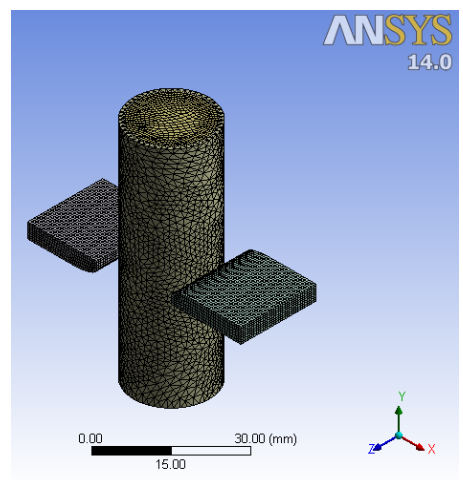

Fig. 2. Miscanthus stalk geometry (a) FEM model meshing (b) [12]

Experimental research was realized using an entire miscanthus stalk, which was subjected to cutting with the help of knifes with different opening angles $\left(30,50^{\circ}\right)$ and different sharpenning edge angles $\left(10,20,30,40\right.$ and $\left.50^{\circ}\right)$. The stalks were harvested from the experimental field of the National Institute of Agricultural Machinery Bucharest (plant height 130-280 cm, base medium diameter 8-12.5 $\mathrm{mm}$, top medium diameter 3.5-5.0 mm, number of internodes between 10-15, plant weight 8.9-37.6 g, moisture content 8.8-9.0\%). For cutting tests a mechanical testing apparatus Hounsfield H1KS fitted with a cell force of $1000 \mathrm{~N}$ was used, the blade cutting speed of $500 \mathrm{~mm} \cdot \mathrm{min}^{-1}\left(8.3 \cdot 10^{-3} \mathrm{~m} \cdot \mathrm{s}^{-1}\right)$. The cutting process was done for 7 internodes of the stalks, numbered from the bottom to the top, where the cutting is higher. The experimental setting of the knife connected to the loading cell of Hounsfield equipment used for testing is presented in Fig. 3. Also, it must be said that the material samples were placed on the support plate, so that the force applied by the cutting knife was at the middle of the specimen. While experimental testing the steel blade passes through a slot, thus applying the cutting force on the miscanthus samples.

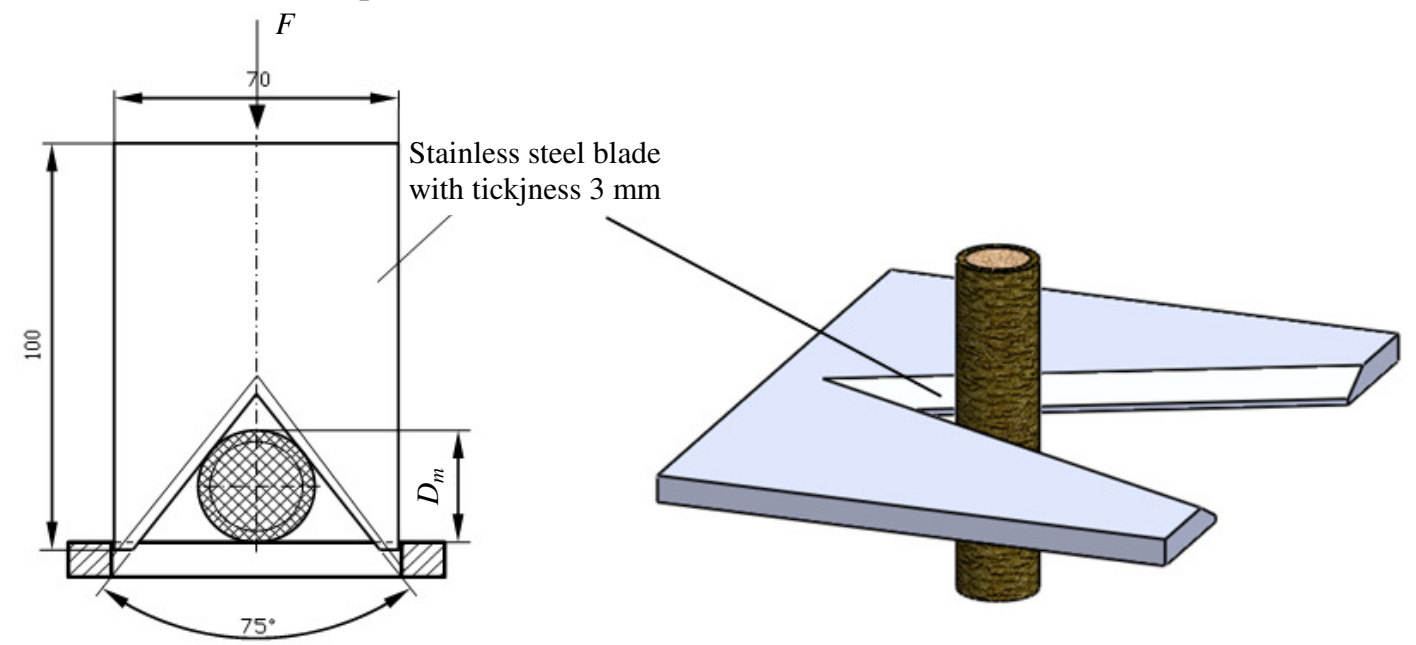

Fig. 3. Device cutting diagram (cutting knife and plant stalk experimental setting) [12] 


\section{Results and discussion}

Based on literature experimental data, the simulation of the plant cutting process with straight knives was realized. The processing was done on the entire model, considering the asymmetry of the loading system considered. The plant loading system was simulated maintaining the surfaces fixed from the inferior part of the plant (rigid fixing) and the working surface was subjected with a force relative to each loading phase. Because it was done on the entire model, the loading forces presented in the model are equal in magnitude, but on opposite directions. Studies were done for miscanthus stalks for the shear cutting forces between $100 \mathrm{~N}$ and $250 \mathrm{~N}$. From the model structural analysis the mesh model resulted in 64750 elements and 159220 joints. The mesh model results from the model structural analysis for both blades as well as for the shell and kernel are presented in Table 1.

Table 1

Statistic of used model solid part element network

\begin{tabular}{|l|l|l|l|l|l|}
\hline \multicolumn{5}{|c|}{ Statistic mesh } & \multicolumn{1}{c|}{ Total } \\
\hline Object Name & Shell & Blade 1 & Kernel & Blade 2 & - \\
\hline Nodes & 64390 & 4622 & 100381 & 3379 & 159220 \\
\hline Elements & 37255 & 2453 & 23256 & 1786 & 64750 \\
\hline
\end{tabular}

For FEM simulation a stationary analysis was chosen. The system equation numerical method solver was PCG type (Preconditioned Conjugate Gradient) with a Newton-Raphson type, an interative solver used in general for unbalanced problems. The entire determination was done with double precision by imposing the converging criteria of $10^{-4}$ to all residue in order to reduce numerical errors. It took aproximatly 175 iterations, so the solution to converge with the tolerance imposed after a processing time of about 48 hours.

Analyzing Fig. 4.a, the way the first stress appears in the plant during testing can be seen. It can be observed that the maximum values obtained are at the shear stress of $97.514 \mathrm{MPa}$. Regarding the energy consumed for the cutting process, in Fig 4b. we can see the maximum energy of $39.66 \mathrm{~mJ}$. Also, the maximum stalk deformation on the $\mathrm{OZ}$ cutting axis could be observed, which was about $2.5 \mathrm{~mm}$, with a gradual distribution towards the middle of the section. From the simulation analysis the importance of correctly following all the FEM analysis procedures resulted, being necessary to verify the simulation data obtained with the experimental determinations. The cutting process with Vshape blades and different opening angles is very different fom the cutting process with a simple blade, disregarding the blade obliquity from the horizontal (the plant being on this direction and perpendicular on the blade plan).

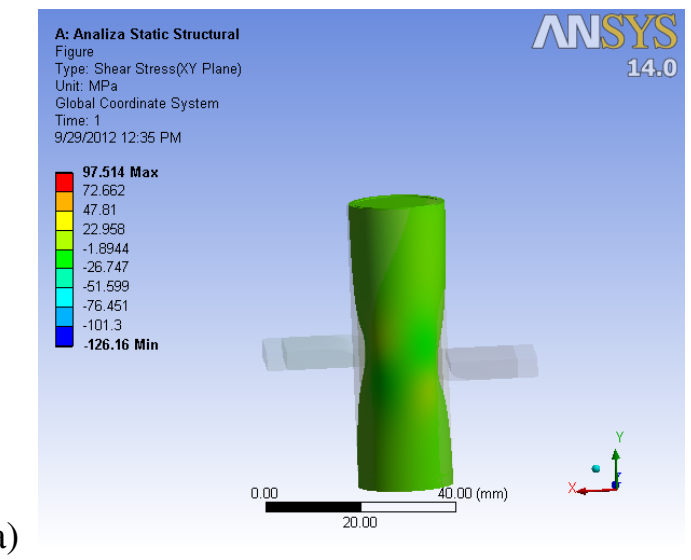

b)

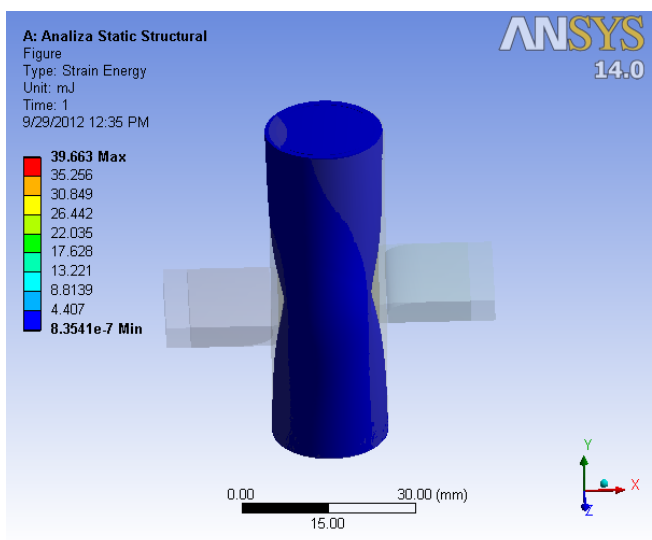

Fig. 4. FEM Simulation results: a - values obtained during the first stresses applied on the stalk; b - deformation energy values [12])

Experimental research with $\mathrm{V}$ type blades highlighted the cutting curves relatively similar to other speciality theories for each of the knives being analyzed. Because the material samples had large variations of the diameter, its mass and the lignocellulose plant shell, crushing, shear and cutting force, disregarding the blade cutting angle the variations corresponding to the yield point were also in large limits. For plants subjected to testing, the medium cutting force decreased from about $600 \mathrm{~N}$ at the 
bottom internode to about $110 \mathrm{~N}$ for the $7^{\text {th }}$ internode, the variation curve presenting a decreasing linear variation. Also, from the experimental research it could be concluded that the cutting force for the lower internodes varies in larger limits comparative to the top internodes of the same plant [12]. It is hard to distinguish in Fig. 5 the three characteristic areas, such as in Fig. 1, but it can be observed that the cutting force increases up to a maximum with some fluctuations, which proves the fact that first the plant in compressed and then cut. The cutting process does not stop, when the force reaches the maximum point, it continues afterwards, but the force decreases on the second part, when the deformation process increases. In Fig. 5 the force - deformation curves resulted during the experimental testing are presented for four different sharpening edge angles: a) force - deformation curve for $10^{\circ}$ sharpening edge angles, b) force - deformation curve for $20^{\circ}$ sharpening edge angles, c) force - deformation curve for $30^{\circ}$ sharpening edge angles, d) force - deformation curve for $40^{\circ}$ sharpening edge angles.
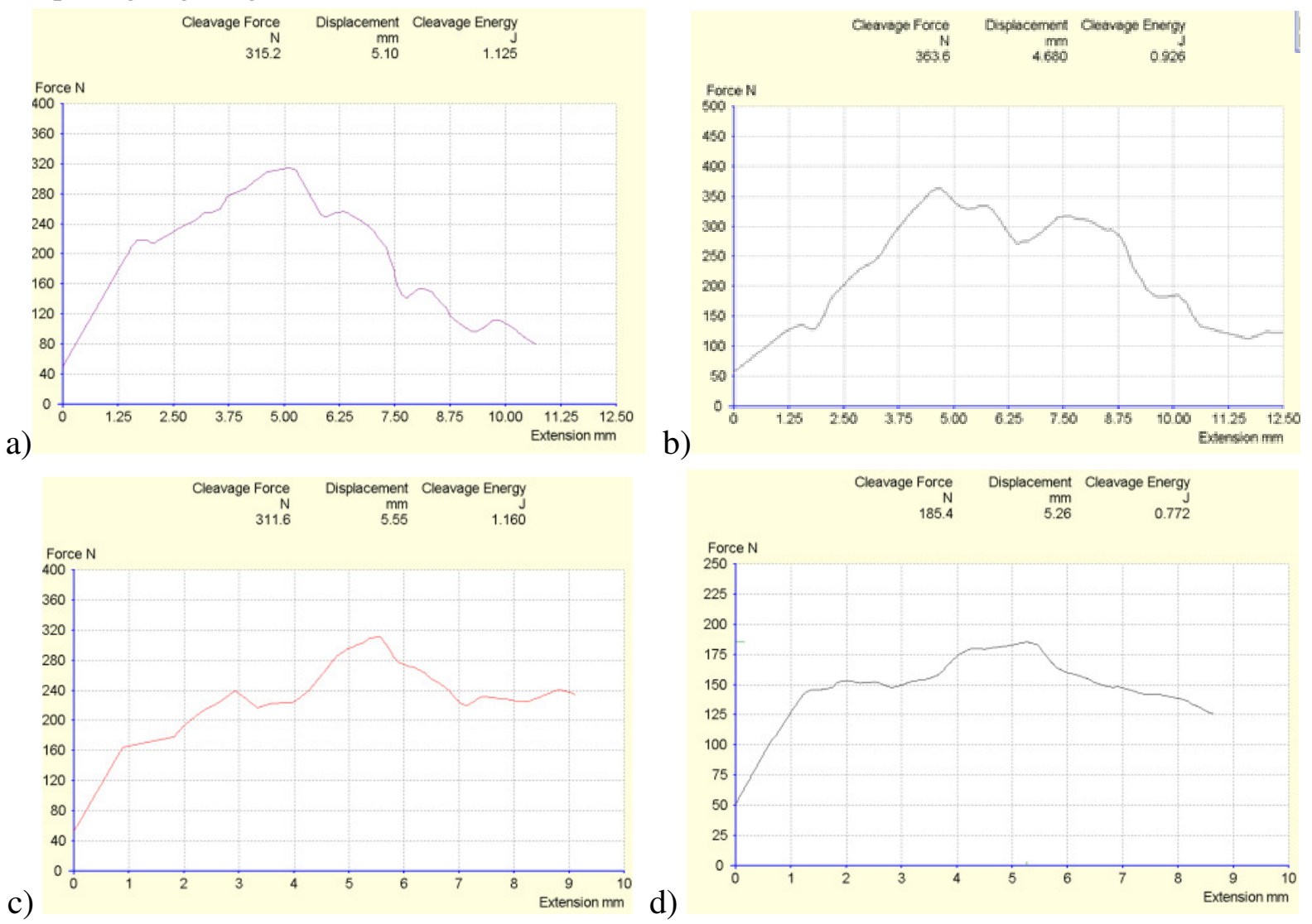

Fig. 5. Force - deformation curves resulted after cutting tests with $\mathbf{V}$ type knives and 50 degrees opening and different sharpening edge angles (sharpening edge angles: $\left.\mathrm{a}-10^{\circ} ; \mathrm{b}-20^{\circ} ; \mathrm{c}-30^{\circ} ; \mathrm{d}-40^{\circ}\right)$

The stalk used for testing had a medium diameter of $8.74 \mathrm{~mm}$. After the experimental research the plant cutting force variation considering the knife sharpening edge angle could be determined. It can be said that minimum values for the cutting force were obtained for a sharpening edge angle of 30 and 40 degrees, while for a sharpening angle of 20 degress the medium force values were about $464 \mathrm{~N}$ and for the sharpening angle of 50 degrees the medium cutting force was about $424 \mathrm{~N}$, all determinations being at the blade cutting speed of $500 \mathrm{~mm} \cdot \mathrm{min}^{-1}$.

It is necessary to say that the shear cutting stress (cutting force/stalk section area) had medium values between $5 \cdot 10^{6} \mathrm{MPa}$ for the internode 1 and $3.8 \cdot 10^{6} \mathrm{MPa}$ for the internode 5, a decrease in the values to the top of the plants. Also, the shell thickness was an important factor of influence on the cutting force having the values between $1.9-2.0 \mathrm{~mm}$ for the internode 1 to $1.05-1.10 \mathrm{~mm}$ for the internode 7 , decreasing to the top of the plant. Thus, the cutting force, stress and cutting energy are very much influenced by the reminded factors and also by the plant's moisture content with large variations in values, disregarding the cutting method applied and the cutting blade characteristics. 


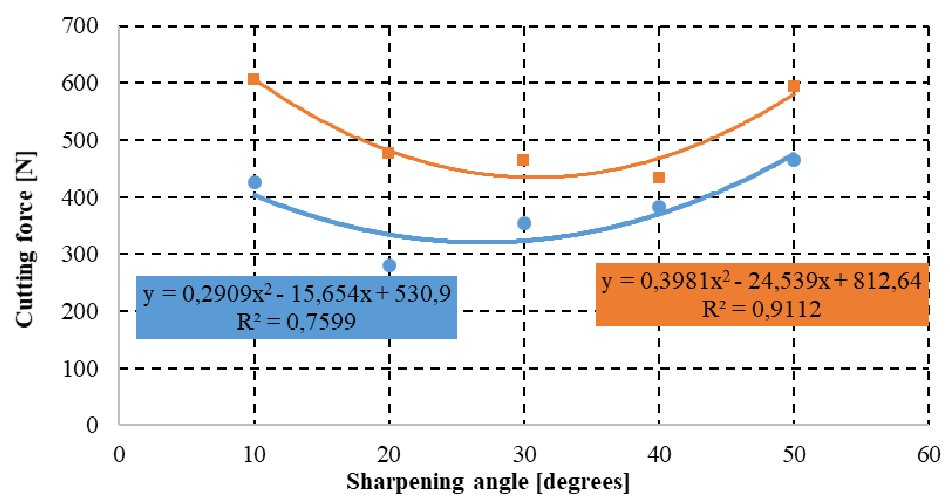

a)

- 50 degrees $\quad 30$ degrees

30 degrees blade opening angle

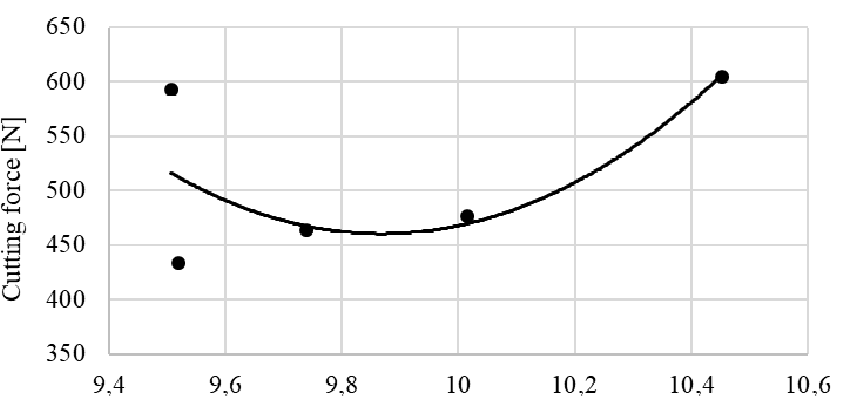

b)

Medium diameter $[\mathrm{mm}\rceil$

Fig. 6. Cutting force variation considering sharpening edge angles of $V$ type knives (a), cutting force variation considering medium diameter of material samples for $V$ type knife and opening of 30 degrees (b)

\section{Conclusions}

1. 3D advanced FEM analysis is an important instrument in making detailed investigations referring to cutting behavior in connection to cutting blades. In our simulation, the shear force was 97.514 MPa considering all the process inputs.

2. The maximum deformations of the miscanthus stalk highlighted by the simulation program was on the OX axis of $7.08 \mathrm{~mm}$, on the OY axis of $0.74 \mathrm{~mm}$ and for the $\mathrm{OZ}$ axis of $2.5 \mathrm{~mm}$, which happens in a real cutting process.

3. The cutting force for the knife speed of $500 \mathrm{~mm} \cdot \mathrm{min}^{-1}$ expressed that an optimum sharpening edge angle would be 30 degrees, considering that the medium force values registered were for this edge for $\mathrm{V}$ type knives with an opening of 30 or 50 degrees. The differences between the values obtained from experiments and simulation are due to the knife model used, different from one case to another.

4. The cutting force resulted after the experimental research showed a medium force value of about $424 \mathrm{~N}$ for the sharpening angle of 50 degrees. Also, the experimental research indicated an increasing variation of the cutting force, when considering the medium stalk diameter.

5. The results obtained and presented in the paper prove that the simulation analysis method for the biomass cutting process can be used successfully, identifying that the experimental research on the field was in accordance with the process simulation.

\section{Acknowledgements}

The work has been funded by the National Grants GNaC 2018 ARUT, Internal Research Grant, through the Financial Agreement 06/15.10.2018. 


\section{References}

[1] Mohsenin N. Physical Properties of Plant and Animal Material. Gordon and Breach publichers Inc., Amsterdam, The Netherlands, 1986.

[2] Igathinathane C., Womac A.R., Sokhansanj S. Effect of Angle Cut on Corn Stalks Mechanical Cutting Strength and Energy. An ASABE Meeting Presentation, Paper number 1008644, Presentation written for presentation at the 2010 ASABE Annual, 2010;

[3] Shastri Y.N., Miao Z., Rodríguez L.F. etc. Determining optimal size reduction and densification for biomass feedstock using the BioFeed optimization model. Biofuels, Bioproducts and Biorefining, vol. 8, issue 3, 2014, pp. 423-437.

[4] G. Ferro, R. Minciardei, E. Podesta. etc. An optimization model for the sizing of the biomass plants supply chain, ScienceDirect ELSEVIER, IFAC conference Papers Online 51-5, 2018, pp 114-119.

[5] Mani S., Tabil L.G., Sokhansanj S. An overview of compaction of biomass grinds. Powder Handling and Processing, vol. 15, issue 2, 2003, pp.160-168.

[6] Samson, R., Mani S., Boddey R., etc. The potential of C4 perennial grasses for developing a global bioheat industry. Critical Reviews in Plant Science, vol. 24, 2005, pp. 461-495.

[7] Wang W.W., Li J.C., Chen L.Q. etc. Effects of key parameters of straw chopping device on qualified rate, non-uniformity and power consumption. Internationl Journal of Agric \& Biol Engineering, vol. 11, 2018, pp. 122-128.

[8] Kovács Á., Zwierczyk P.T. Coupled DEM-FEM simulation on maize harvesting, Proceedings 32nd European Conference on Modelling and Simulation OECMS Lars Nolle, May 22-25, 2018, Wilhelmshaven, Germany ,pp. 405-411.

[9] Kovács Á., Kerényi GY. Comparative analysis of different geometrical structures of discrete element method (DEM) for fibrous agricultural materials. 4th CIGR International Conference of Agricultural Engineering, June 26-29, 2016, Aarhus, Denmark, pp. 1-8.

[10] Kemper S.; T. Lang; L. Frerichs. "The overlaid cut in a disc mower - results from field tests and simulation." Landtechnik, vol. 69, 2014, pp. 171-175.

[11]Cristian S., Voicu E., Dragoş M., etc. Technology for promotion in Romania of energy crop Miscanthus as renewable resource to increase energy competitiveness in independence purposes, Scientific Papers INMATEH Bucharest, vol 29, No. 3, 2009, pp. 10-15.

[12] Moiceanu G. Research regarding energetic plant behavior during mechanical operation of cutting and grinding, $\mathrm{PhD}$ Thesis 2012. 